\title{
Tub toys and computers: A review of Odd Perceptions
}

\author{
DENNIS R. PROFFITT \\ University of Virginia, Charlottesville, Virginia
}

\section{Odd Perceptions}

By Richard L. Gregory. 1986, London: Methuen

(Paperback, 1988, New York: Routledge). 230 pp.

Odd Perceptions is a collection of essays by Richard $\mathrm{L}$. Gregory, one of the most prominent visual scientists of our time. Most of the essays are elaborations on editorials that were originally published in Perception, the journal for which Gregory has served as editor since its inception in 1972. The essays cover such a variety of topics that it is impossible to categorize their content or even define boundaries for their range. For example, Gregory writes about bath toys, computers, conjuring, ESP, intelligence, illusions, hieroglyphics, and reflections on a nearly missed automobile accident. Despite this diversity, the essays are unified by recurrent themes, and by the good humor and intelligence of their author.

Repeatedly, throughout the essays, Gregory expresses fascination with what he finds odd and peculiar. For him, the hidden mechanisms of human perception reveal themselves most clearly when our phenomenal experience is at odds with objective reality. Consider the following:

\footnotetext{
I keep two frogs in my bath, and also ducks and a small fish. They wind up, working by waterproof clock. May I commend clockwork frogs and fishes to ethologists and all who study animal, and also human, behaviour? They are wonderful for revealing and calibrating the observer'sincluding one's own-projections of intelligent intention into the world of moving things. (p. 13)
}

That the motions of windup toys evoke a sense of animacy reveals that our perceptions of intentionality are equivo$\mathrm{cal}$. It seems that the perceptual rules that allow us to accurately perceive the intentions of people and animalsfor example, recognizing the motives inherent in a child's game of tag-also force us to see similar intent in the motions of tub toys that coincidentally manifest motions that, to us, appear similar.

Odd perceptions engender curiosity and thereby motivate inquiry into the mechanisms of perception. In most

Correspondence concerning this review should be addressed to Dennis Proffitt, Department of Psychology, Gilmer Hall, University of Virginia, Charlottesville, VA 22903-2477. everyday situations, our perceptual processes seem transparent, and the phenomenal world is uncritically accepted as being reality. Introductory psychology textbooks typically emphasize illusions in their perception chapter as a means to engage students' interest. Otherwise, students often have a difficult time comprehending why there is a need to study perception at all. After all, common sense suggests that the world reveals itself in perception pretty much as it is.

Apart from the advantage that odd perceptions have in motivating an appreciation for perceptual studies among the naive, there is a far deeper issue to be considered in defining the status of illusions in the visual sciences. Odd perceptions are so surprising because most perceptions are relatively veridical, at least when evaluated from a functional perspective: the perceptual guidance of most everyday actions is exceedingly good. From the mundane achievements of simple pattern recognition to the awesome feats of skilled athletes, the visual system holds claim to accomplishments that no visual scientist claims to understand.

Will visual research be furthered best by focusing on the perceptual system's veracious abilities or upon its surprising peculiarities? This question is at the core of a controversy that separates two theoretical schools of thought in perceptual psychology. The answer seems not to be in the immediate offing, as proponents of each school continue today to argue at cross purposes with each other, to little effect. On the other hand, some progress toward reconciling the oddness of perceptions with the effectiveness of actions may be achieved through investigations of perception and action coupling. Consider an example.

When people first visit San Francisco, they cannot help but be struck by how incredibly steep are some of the roads that they encounter. When asked to estimate the slopes of these hills, people provide erroneous estimates in the neighborhood of $45^{\circ}-75^{\circ}$ (informally collected anecdotal evidence). In fact, the steepest road is no more than $15^{\circ}$. Evidence exists that this is a general finding (Ross, 1974): when approaching a large incline, such as a hill, people grossly overestimate its slant.

Given that people misperceive the slant of hills, why do they not evidence this misperception when walking up 
them? The answer to this question is that accurate perceptions of environmental properties are not required for effective control of actions. As long as perceptual attributes covary with environmental dimensions, control will not reflect underlying misperceptions.

For expository purposes, consider how control behaviors are learned in a situation such as flying a helicopter at low altitudes over a hill. (Here, the actual control mechanisms are oversimplified.) Suppose that the novice pilot misperceives the slant of a hill to be $60^{\circ}$ when, in fact, its inclination is $15^{\circ}$. In order to maintain the desired altitude relative to the hill, the pilot must learn to couple the appropriate control responses to what is perceived. To put the matter simply, the pilot must learn to pull back on the stick by some amount, given that a hill of some perceived slant is approaching. Through learning, the pilot will come to couple the appropriate control responses to the misperception of slant. It does not matter that slant is misperceived, since control responses have been acquired in the context of this misperception, and the misperception covaries with distal slant.

Gregory's emphasis on what is odd about perception is a common but not universally held perspective among visual scientists. Illusions and veridical perceptions are both inherent in phenomenal experience. What is not clear is whether illusions are the product of unconscious inferences gone astray, or of sufficing mechanisms in which illusory mediators are best understood in the context of their practical consequences.

Thus, although it is true that tub toys may appear to have animate motions, they are rarely if ever misperceived as being anything other than what they are-simple machines. If only this were true of computers.

Another recurrent theme in Gregory's essays is his perception of unlimited possibilities for computers to shed light on the most fundamental issues of mind. Again and again, he asks us to contemplate what it will be like when computers achieve perceptual competencies, and thereby acquire their own odd perceptions.

The expectation that computer models will someday emulate human abilities is grounded solely in a faith in the technology and not in its actual achievements. There is little question that computers are useful tools for modeling perceptual competencies; however, there is no evidence that computer vision models will someday rival the achievements of the human perceptual system. Current computerized pattern-recognition systems appear competent only when the domain of objects they must identify are stereotypic members of some finite set of alternatives. It is common to refer to such domains as "toy worlds." There exist no general pattern-recognition models capable of categorizing natural ill-defined images drawn from an indefinite set of alternatives. Even the recognition of human handwriting, in which the categorical alternatives are confined to the 26 letters of the alphabet, far exceeds the capabilities of the most advanced pattern-recognition machine. The ability to construe order in the variety and novelty of everyday experience is, of course, the hallmark of human perception.

Gregory's musings about consciousness in intelligent machines is especially irksome given that he makes no attempt to define consciousness. Might it arise from the activity of thinking machines, or might it be a prerequisite of thinking? Assuming that conscious machines are, indeed, possible, there appear to be two alternatives for the spontaneous ontogenesis of their minds. One would be that consciousness is a by-product of thinking machinesbrains and tomorrow's computers-and that it is only an odd accompaniment to their activity. The second alternative is that consciousness is an emergent property of machines that can think with a certain degree of complexity and, perhaps, speed. Here, consciousness may be a functional component of thought and not merely an attendant phenomenon. On the other hand, it may be that human competencies are unachievable without consciousness, and that an understanding of consciousness must precede a successful technological modeling of human perceptual abilities.

As with all speculations of this sort, predications of "maybe" are best echoed with "maybe not." Computer technologies may someday be able to realize artificial minds. Maybe not. Computers may be like tub toys, the motions of which suggest an animacy that can never be realized in their mechanical simplicity.

In the last essay of the book, Gregory notes the danger of seeking models of mind in the most advanced technologies available. Descartes, for example, advanced the current understanding of neural activity by modeling it on the latest plumbing achievements of his time; however, this hydraulic model proved, of course, to be an untenable one. Likewise, attributing the possibility of conscious states to computers may be viewed from a historical perspective as the projection of our own "intelligent intentions" onto the activities of the technology of which we are most proud. However, this qualification comes too late, at least for this reviewer, who had lost patience with Gregory's musings about thinking machines quite early in the book.

In summary, Odd Perceptions is a collection of diverse essays, unified primarily by the fact that their topics have drawn the interest of the lively mind of Richard Gregory. The essays reflect his predilections and wit as much as they draw upon his deep and broad knowledge of perceptual issues. But beware. Although not unique, Gregory's biases should not be accepted as the shared wisdom of the field. As when observing his bath toys, Gregory often reports seeing more oddities in events than do the rest of us.

\section{REFERENCES}

Ross, H. E. (1974). Behavior and perception in strange environments. London: Allen \& Unwin. 